\title{
A Case Report of Effect of Physical Therapy on Temporomandibular Joint Dysfunction Following Mandibular Symphyseal Fracture
}

\author{
Mohammad Reza Asadi 1,* (D), Gholamreza Hajvalie ${ }^{2}$ \\ ${ }^{1}$ Assistant Professor, Department of Physical Therapy, School of Rehabilitation Sciences, Hamadan University of Medical \\ Sciences, Hamadan, Iran \\ ${ }^{2}$ MSc in Physical Therapy, School of Rehabilitation Sciences, Hamadan University of Medical Sciences, Hamadan, Iran \\ * Corresponding Author: Mohammad Reza Asadi, Department of Physical Therapy, School of Rehabilitation Sciences, \\ Hamadan University of Medical Sciences, Hamadan, Iran. Email: reza.asadi21@yahoo.com
}

Received: 06.05.2019

Accepted: 19.08 .2019

How to Cite this Article: Asadi MR, Hajvalie G. A Case Report of Effect of Physical Therapy on Temporomandibular Joint Dysfunction Following Mandibular Symphyseal Fracture. Avicenna J Clin Med. 2019; 25(2): 125-130. DOI: $10.21859 /$ ajcm. 26.2.125

\section{Abstract}

Background: Temporomandibular joint (TMJ) dysfunction following Mandibular fractures can lead to limitation of jaw movement especially month opening, pain, and muscular atrophy. In this regard, the current study aimed to determine the effect of manual mobilization along with other physiotherapy modalities on the improved function of TMJ in a patient with Mandibular symphyseal fracture.

Case Presentation: A 16-year-old girl with a complaint of severe jaw pain and disability of mouth opening was referred to physical therapy clinic of Besat Hospital affiliated to Hamadan University of Medical Sciences. The patient underwent an open surgical treatment due to mandibular symphyseal fracture. A visual analog scale was used for the measurement of pain intensity and the maximal mouth opening was obtained by measuring the distance of the maxillary and mandibular central incisal edges using a dental ruler scaled in millimeters. Thereafter, physical therapy treatment was administered to both TM joints for five sessions every other day. The physiotherapy sessions included Transcutaneous Electrical Nerve Stimulation (TENS), ultrasound, hot pack, and Maitland manual mobilization techniques. The patient demonstrated significant reduction in pain and improved maximal mouth opening upon the completion of 5-session physical therapy treatment.

Conclusion: Physical therapy treatment which includes physical modalities along with manual mobilization techniques may be an effective method in the management of TMJ dysfunctions following mandibular symphyseal fracture leading to faster improvement in the function of this joint.

Keywords: Manual Therapies, Physical Therapy Modalities, Temporomandibular Joint Disorders 
doI: $10.21859 / \mathrm{ajcm} \cdot 26.2 .125$

\title{
كزارش موردى از تأثير فيزيوترايى بر اختلالات مفصل تيجكاهى فكى به دنبال شكستى سمفيزيس استخوان منديبل
}

\author{
محمدرضا اسدى ا." iD ، غلامرضا حاج وليئىى \\ ' استاديار، كروه فيزيوترايى، دانشكده علوم توانبخشى، دانشگاه علوم يزشكى همدان، همدان، ايران

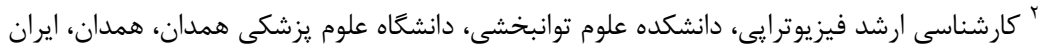

*ق نويسنده مسئول: محمدرضا اسدى، گروه فيزيوترايى، دانشكده علوم توانبخشى، دانشگاه علوم يزشكى همدان، همدان، ايران.

ايميل: yahoo.com

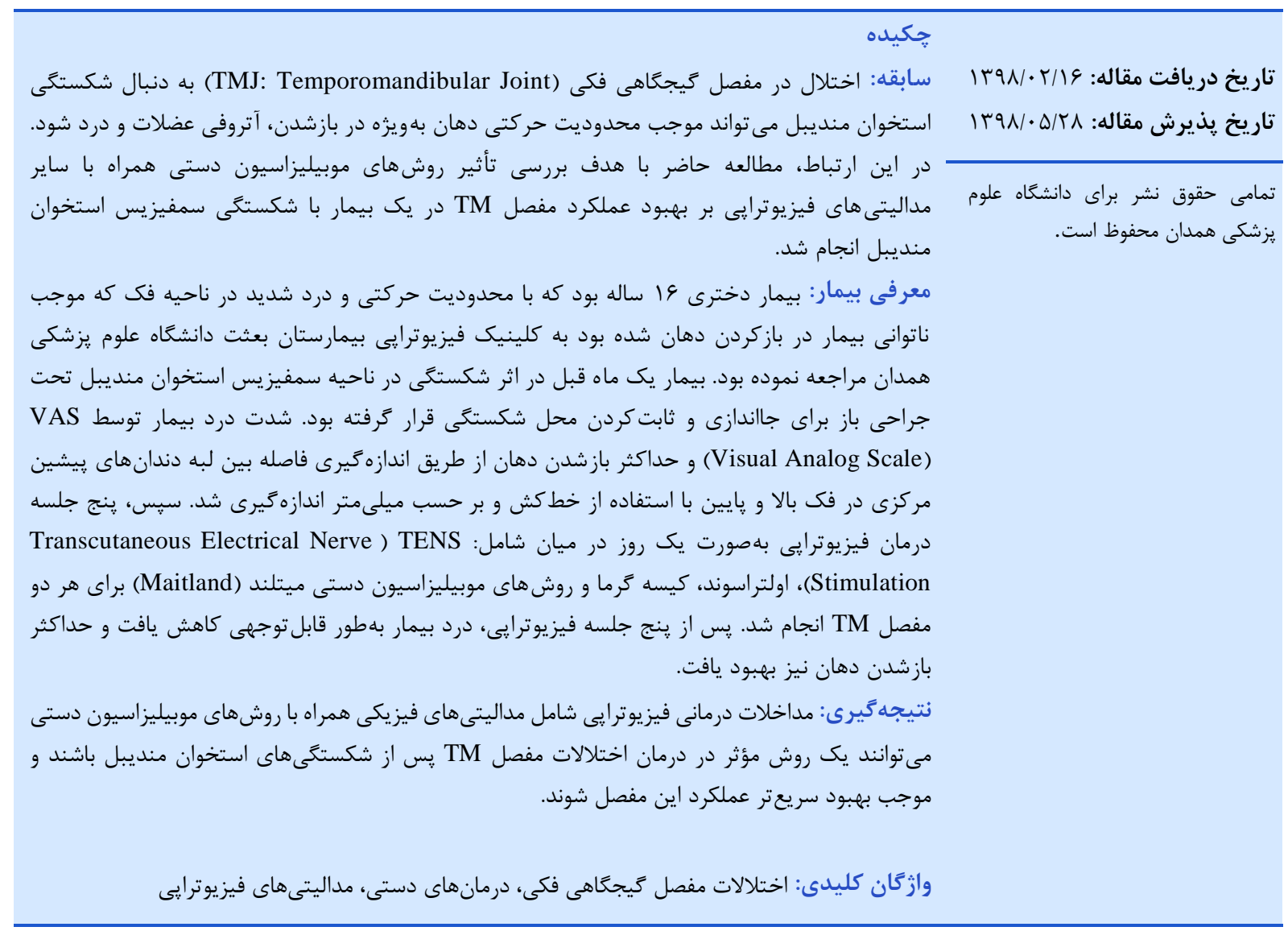

$$
\text { و كيفيت زندى را كاهش دهند. }
$$

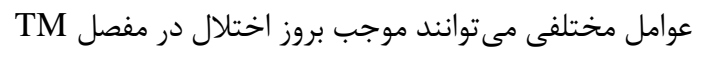

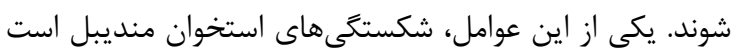

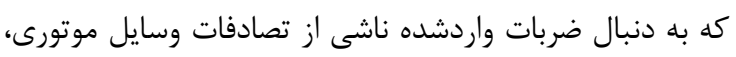

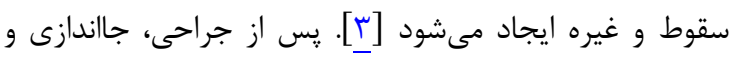

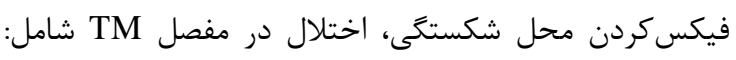

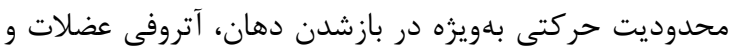

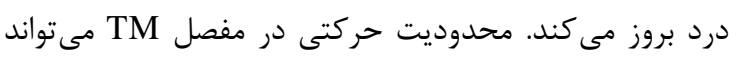

مفصل كيجگًاهى فكى (TMJ) يك مفصل سينوويال است كه بين دو استخوان منديبل و تميورال جمجمه شكل مئى ميرد. اين مفصل عملكرد مهمىى در فعاليتهاى عملكردى روزمره شامل:

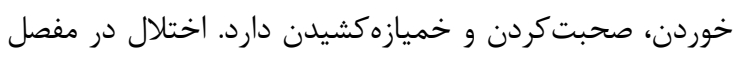

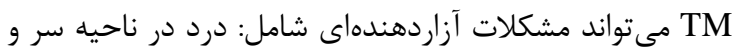
كردن، محدوديت در حركات فك مانند باز و بستهكردن دهان،

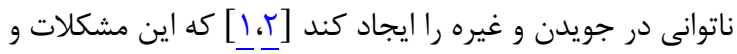
ييامدها مى توانند بر زندگى فردى و اجتماعى بيمار تأثير كذاشته 
براساس مقياس VAS در حال استراحت، بَ و در هنگَام تلاش

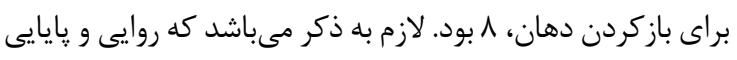

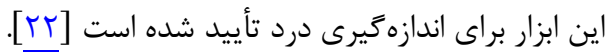

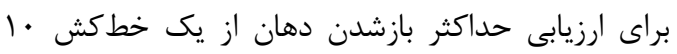

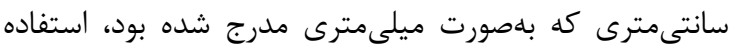

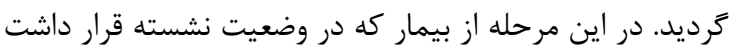

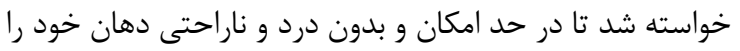

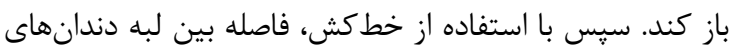

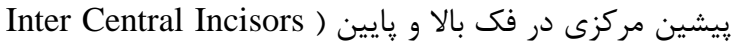

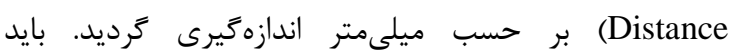
خاطرنشان ساخت كه روايى و يايايى اين شيوه براى اندازهيرى

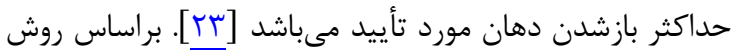

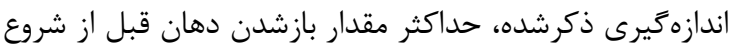

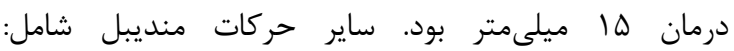
lateral deviation و نيز محدود شده بود؛ اما با توجه به اينكه روش معتبرى براى اندازهخيرى اين

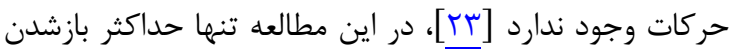
دهان مورد ارزيابى قرار حرفت.

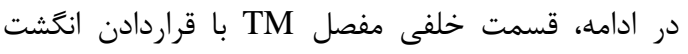

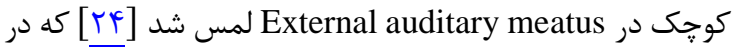
حين باز و بسته كردن دهان، تندرنس وجود داشت. براساس نتايج

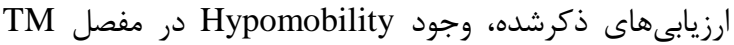

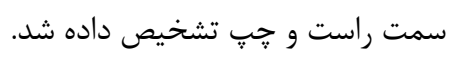

در راستاى انجام اين مطالعه، بيمار زينج جلسه درديص درمان

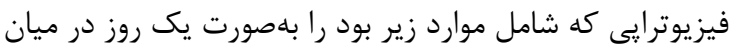

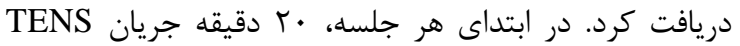
از نوع (Transcutaneous Electrical Nerve Stimulation) نرمال (استيمولاتور 710 P Plus نوين، شركت نوين، ايران) با

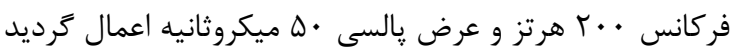

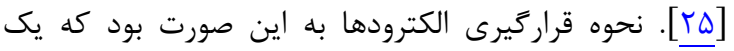

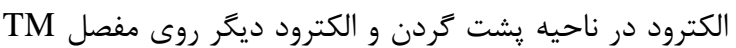

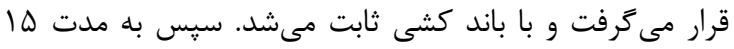

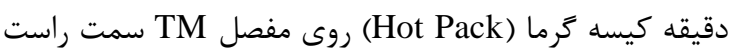

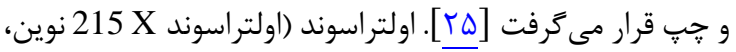

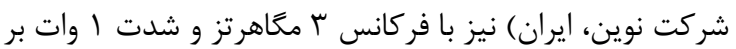

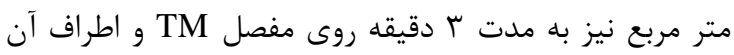

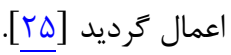

در اين مطالعه روشهاى موبيليزاسيون ميتلند براى مفصل Anterior و شامل: Inferior Glide ، Glide به منظور افزايش تحرك كنديل منديبل انجام شد [عب]. براى موبيليزاسيون مفصل TM جِ، بيمار در وضعيت سورِين

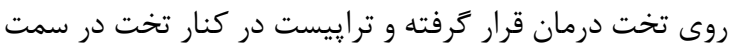

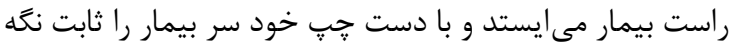

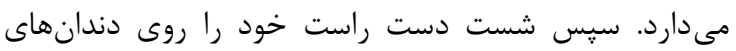

ناشى از التهاب مفصلى يا همارتروز و يا بى حركتى مفصل يس

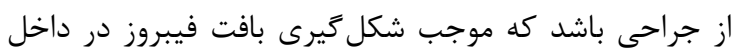

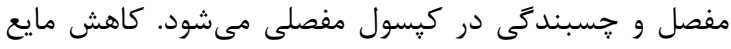

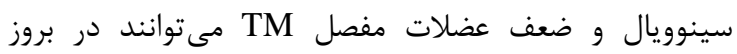

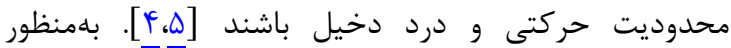

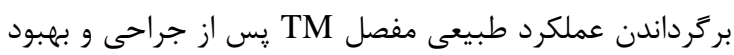

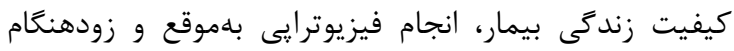

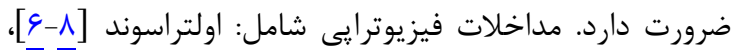

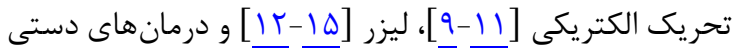

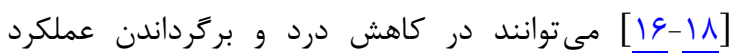

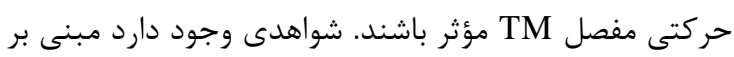

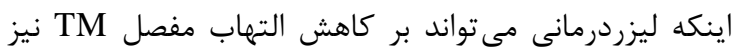

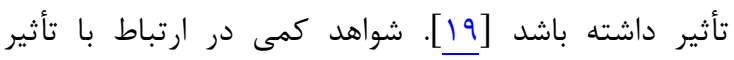

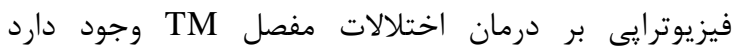

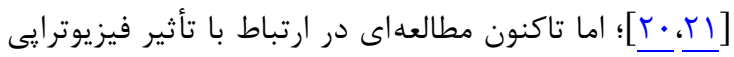
بر اختلالات مفصل TM يس از جراحى شكستكى سمفيزيس

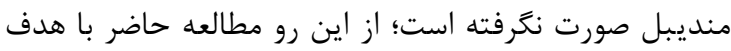

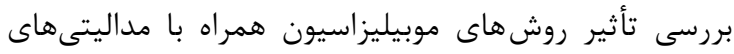

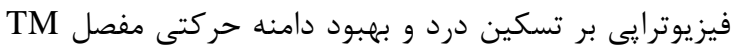

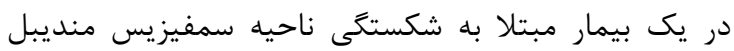

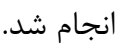

\section{معرفى بيمار}

بيمار يك دختر 19 ساله بود كه با شكايت از درد شديد

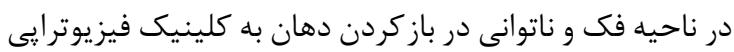

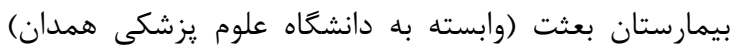

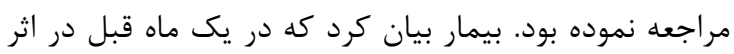

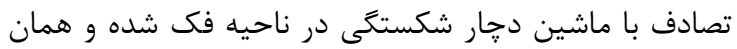

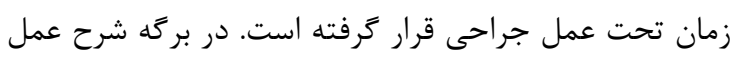

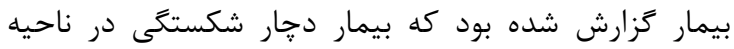

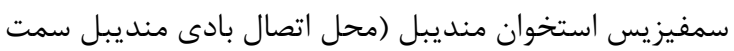

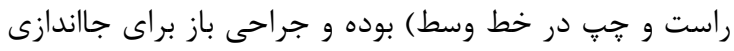

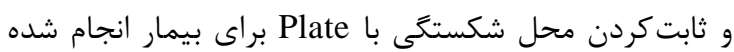
است. بيمار بيان نموده بود كه به دليل درد به بـ ورد محدوديت

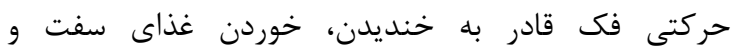
مسواكزندن نمى باشد و در صحبت كردن نيز با مشكلاتى مواجه

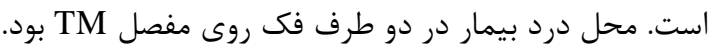

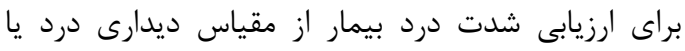

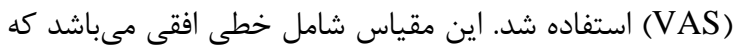

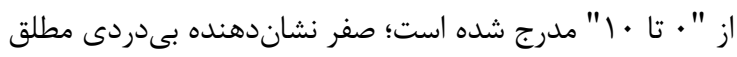

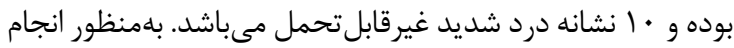

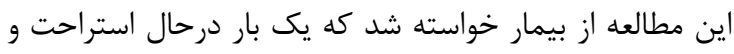
يك بار در هنگام تلاش براى بازكردن دهان با انتخاب عددى بئ بين

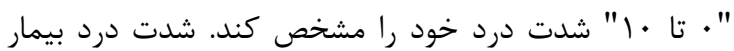


براى بيماران استفاده شد؛ در كروه سوم نيز از تركيب

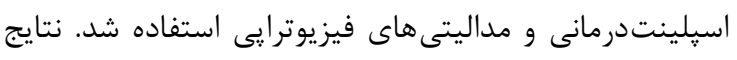

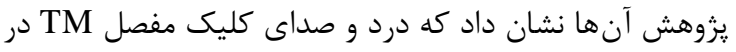

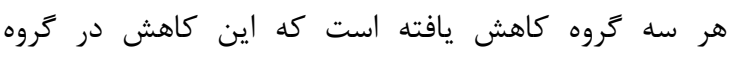

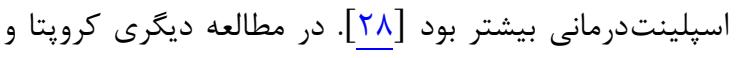

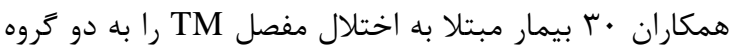

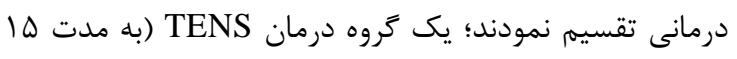

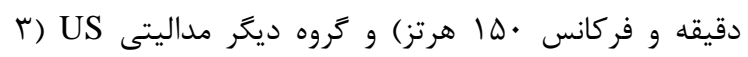

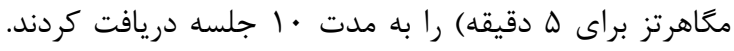

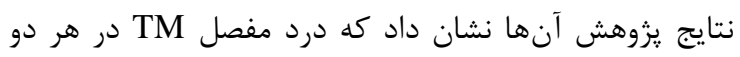

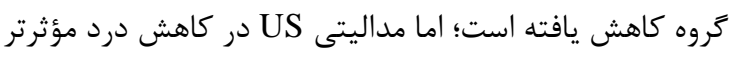

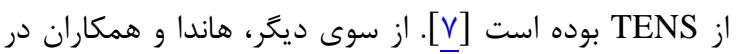

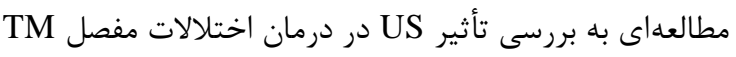

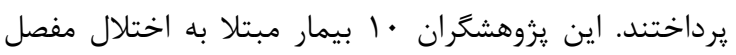

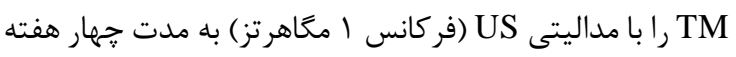

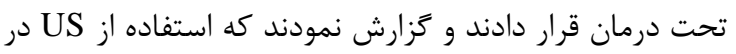

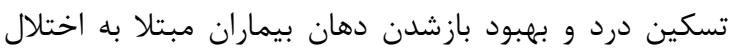

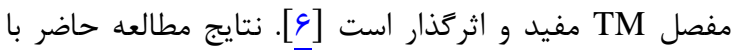

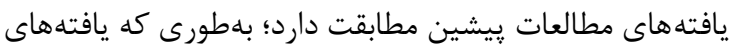

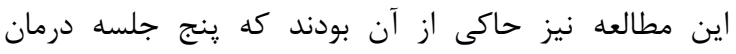

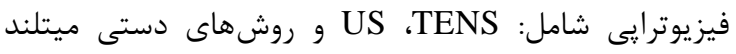

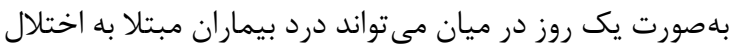

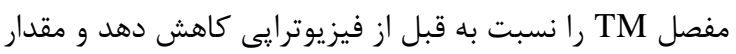

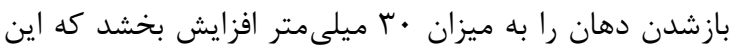

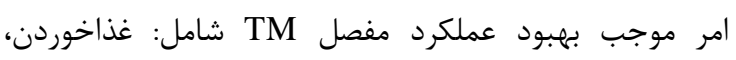
صحبت كردن و خنديدن خواهد شد. در اين مطالعه علاوه بر بردي

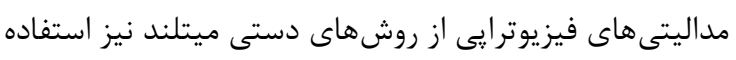

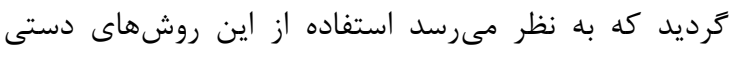

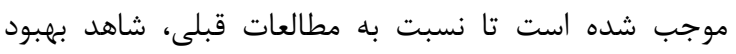
سريعترى در عملكرد مفصل TM باشيم (طى قينج جلسه).

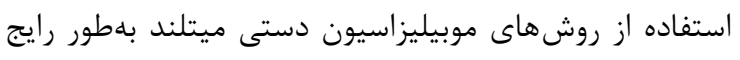

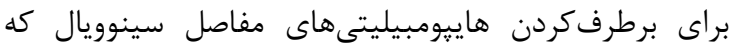

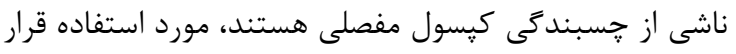

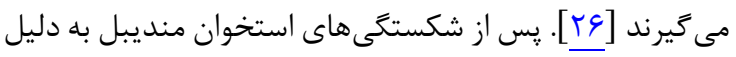

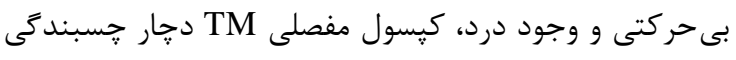

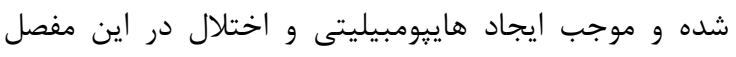

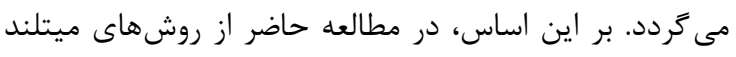

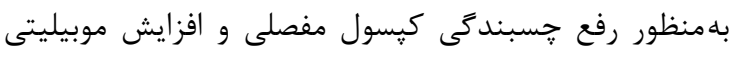

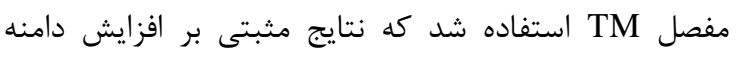

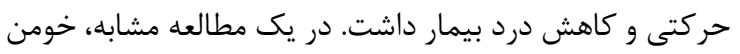

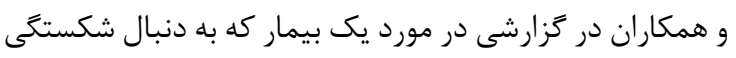

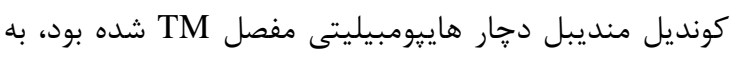

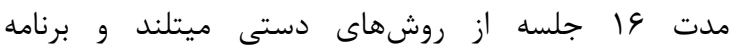
تمريندرمانى در منزل براى افزايش دامنه حركتى و كاهش درئ درد
آسياب بزرى تحتانى (Inferior Molars) سمت جֶٍ بيمار

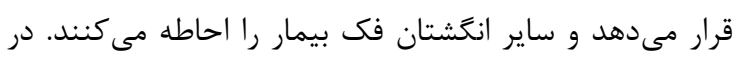

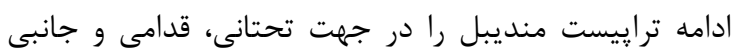

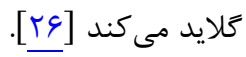

بهمنظور استرج عضلات بالابرنده فك (شامل عضلات Medial Pterygoid و Masseter ،Temporalis آموزش داده شد تا جلوى آينه بنشيند و دهان را بهصورت اكتيو

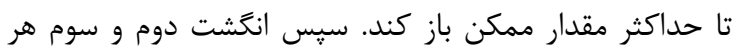

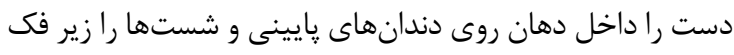

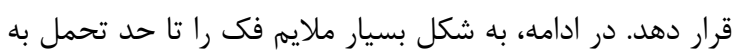

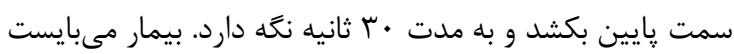

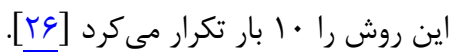

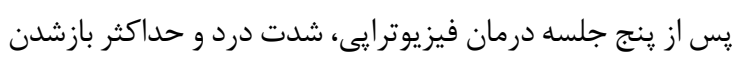

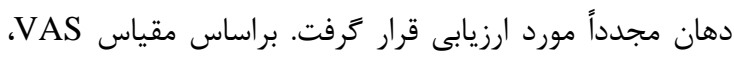

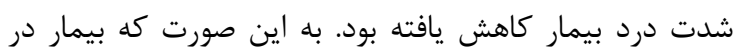

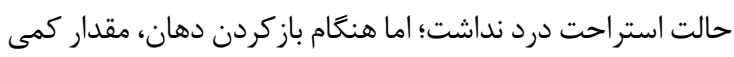

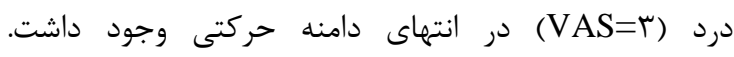

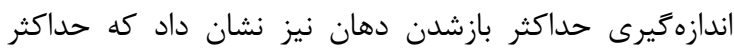

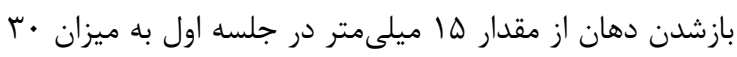

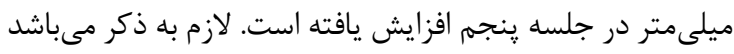

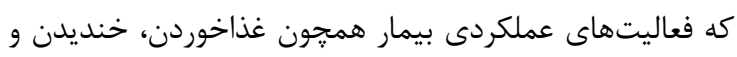

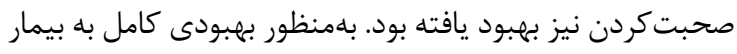

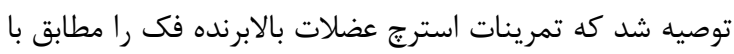

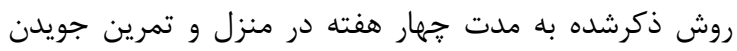

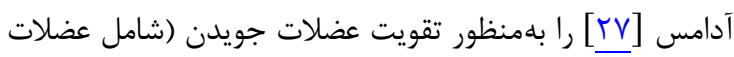
إنجام دمe Masseter ، Temporalis

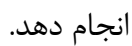

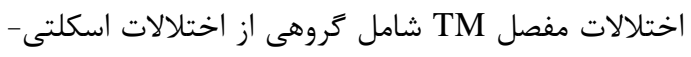

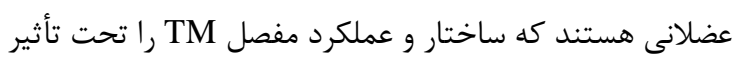

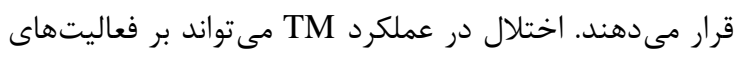

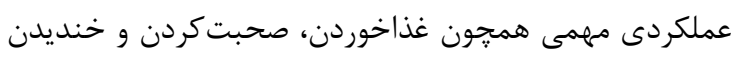

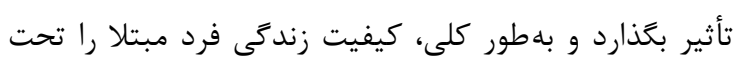

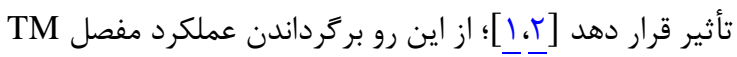

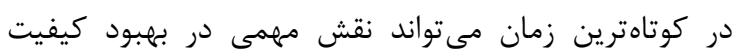

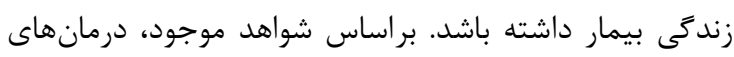

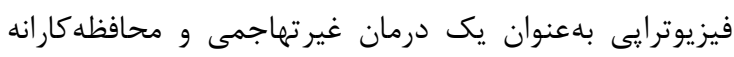

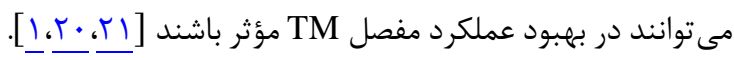

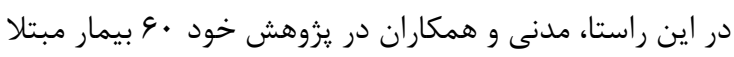

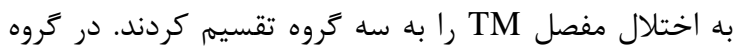

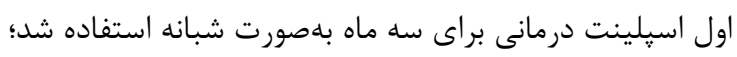

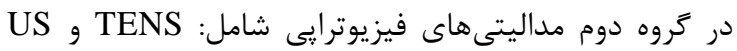

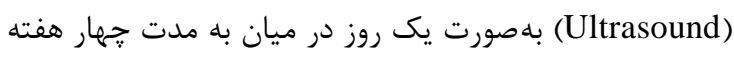




$$
\begin{aligned}
& \text { در كاهش درد، افزايش انعطافيذيرى بافتهاى نرم اطراف } \\
& \text { مفصل TM و در نتيجه بهبود تحرك اين مفصل و تسريع }
\end{aligned}
$$

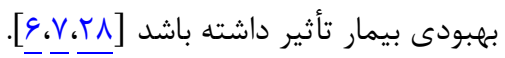

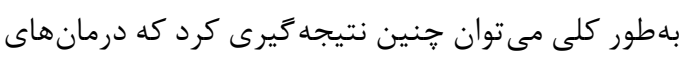

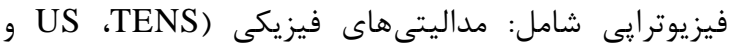

$$
\begin{aligned}
& \text { (Hot Pack }
\end{aligned}
$$

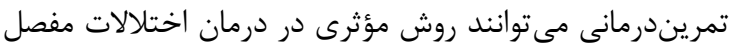

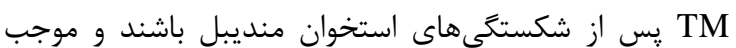

$$
\begin{aligned}
& \text { بهبود سريعتر عملكرد اين مفصل شوند. }
\end{aligned}
$$

\section{REFERENCES}

1. Liu F, Steinkeler A. Epidemiology, diagnosis, and treatment of temporomandibular disorders. Dent Clin North Am. 2013;57(3):465-79. PMID: 23809304 DOI: 10.1016/j.cden. 2013.04.006

2. Goncalves DA, Dal Fabbro AL, Campos JA, Bigal ME, Speciali JG. Symptoms of temporomandibular disorders in the population: an epidemiological study. J Orofac Pain. 2010;24(3):270-8. PMID: 20664828

3. Motamedi MH. A textbook of advanced oral and maxillofacial surgery. Croatia: BoD-Books on Demand; 2013.

4. Chaurasia N, Guan J, Wang X, Sah G. Clinical analysis of changes in function of the temporomandibular joint after open reduction and internal fixation of mandible fracture. Indian J Oral Sci. 2015;6(2):60-4. DOI: 10.4103/09766944.162653

5. Dworkin SF. Research diagnostic criteria for temporomandibular disorders: current status \& future relevance. J Oral Rehabil. 2010;37(10):734-43. PMID: 20529171 DOI: 10.1111/j.1365-2842.2010.02090.x

6. Handa R, Sunil M, Gupta C, Raina A, Khan T, Gulzar A. Efficacy of ultrasound massage therapy as an adjuvant pain control modality in TMDs: a clinical study. J Indian Acad Oral Med Radiol. 2018;30(2)107-9. DOI: 10.4103/jiaomr. jiaomr 5 18

7. Kirupa K, Divya Mary S, Vaishnavi G, Nisha RN, Mercy JR, Jaiganesh G. A comparative study of ultrasound therapy and transcutaneous electrical nerve stimulation in reducing pain for temporomandibular joint disorder. Drug Invent Today. 2019;12(3):515-7.

8. Ucar M, Sarp U, Koca I, Eroglu S, Yetisgin A, Tutoglu A, et al. Effectiveness of a home exercise program in combination with ultrasound therapy for temporomandibular joint disorders. J Phys Ther Sci. 2014;26(12):1847-9. PMID: 25540479 DOI: $10.1589 /$ ipts.26.1847

9. Awan KH, Patil S. The role of transcutaneous electrical nerve stimulation in the management of temporomandibular joint disorder. J Contemp Dent Pract. 2015;16(12):984-6. PMID: $\underline{27018034}$

10. Nunez SC, Garcez AS, Suzuki SS, Ribeiro MS. Management of mouth opening in patients with temporomandibular disorders through low-level laser therapy and transcutaneous electrical neural stimulation. Photomed Laser Surg. 2006;24(1):45-9. PMID: 16503788 DOI: 10.1089/pho. $\underline{2006.24 .45}$

11. Shanavas M, Chatra L, Shenai P, Rao PK, Jagathish V, Kumar SP, et al. Transcutaneous electrical nerve stimulation therapy: an adjuvant pain controlling modality in TMD patients - A clinical study. Dent Res J. 2014;11(6):676-9. PMID: 25540662

12. Chang WD, Lee CL, Lin HY, Hsu YC, Wang CJ, Lai PT. A meta-analysis of clinical effects of low-level laser therapy on temporomandibular joint pain. J Phys Ther Sci. 2014; 26(8):1297-300. PMID: 25202201 DOI: 10.1589/jpts. $\underline{26.1297}$

13. Ahmad RE, Elatief EE, El Sayed WH, Ali RR, Ashour EM, Abdelsamee MY. Effect of conventional therapy and low level laser therapy on pain and limitation of daily functions in patients with temporomandibular joint dysfunction. Int $J$ Physiother Res. 2018;6(2):2797-805. DOI: 10.16965/ijpr.
مفصل TM استفاده كردند و گزارش نمودند كه مداخلات

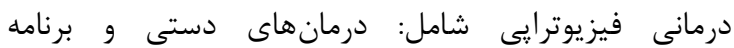

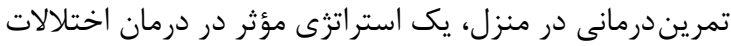

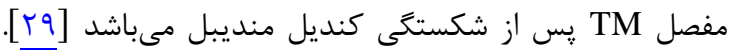

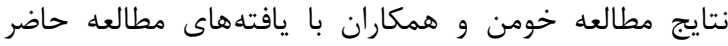

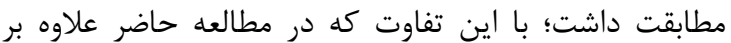

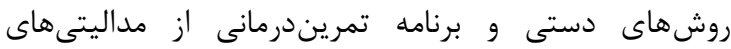

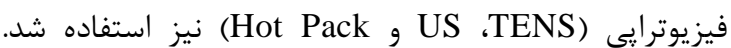
استفاده از اين مداليتى ها همراه با درمانهاى دستى مىتواند

2018.154

14. Sayed N, Murugavel C, Gnanam A. Management of temporomandibular disorders with low level laser therapy. J Maxillofacial Oral Surg. 2014;13(4):444-50. PMID: 26225009 DOI: 10.1007/s12663-013-0544-1

15. Shukla D, Muthusekhar MR. Efficacy of low-level laser therapy in temporomandibular disorders: a systematic review. Natl J Maxillofac Surg. 2016;7(1):62-6. PMID: 28163481 DOI: 10.4103/0975-5950.196127

16. Armijo-Olivo S, Pitance L, Singh V, Neto F, Thie N, Michelotti A. Effectiveness of manual therapy and therapeutic exercise for temporomandibular disorders: systematic review and meta-analysis. Phys Ther. 2016;96(1):9-25. PMID: 26294683 DOI: 10.2522/ptj. $\underline{20140548}$

17. Calixtre LB, Moreira RF, Franchini GH, AlburquerqueSendin F, Oliveira AB. Manual therapy for the management of pain and limited range of motion in subjects with signs and symptoms of temporomandibular disorder: a systematic review of randomised controlled trials. J Oral Rehabil. 2015;42(11):847-61. PMID: 26059857 DOI: 10.1111/joor. 12321

18. Tuncer AB, Ergun N, Tuncer AH, Karahan S. Effectiveness of manual therapy and home physical therapy in patients with temporomandibular disorders: a randomized controlled trial. J Bodyw Mov Ther. 2013;17(3):302-8. PMID: 23768273 DOI: 10.1016/j.jbmt.2012.10.006

19. Barretto SR, de Melo GC, dos Santos JC, de Oliveira MG, Pereira-Filho RN, Alves AV, et al. Evaluation of antinociceptive and anti-inflammatory activity of low-level laser therapy on temporomandibular joint inflammation in rodents. J Photochem Photobiol B. 2013;129:135-42. PMID: 24231378 DOI: 10.1016/j.jphotobiol.2013.10.002

20. Khaled YA, Brennan MI, Napeñas JO. Using physical therapy to treat temporomandibular disorders. A cohort study. J Dent Maxillofacial Res. 2018;1(1):31-5. DOI: 10.30881/jdsomr.00008

21. Melis M. The role of physical therapy for the treatment of temporomandibular disorders. J Orthod Sci. 2013;2(4):1134. PMID: 24987651 DOI: 10.4103/2278-0203.123196

22. Ferreira-Valente MA, Pais-Ribeiro JL, Jensen MP. Validity of four pain intensity rating scales. Pain. 2011;152(10):2399404. PMID: 21856077 DOI: 10.1016/j.pain.2011.07.005

23. Walker N, Bohannon RW, Cameron D. Discriminant validity of temporomandibular joint range of motion measurements obtained with a ruler. J Orthop Sports Phys Ther. 2000; 30(8):484-92. PMID: 10949505 DOI: 10.2519/jospt. 2000.30.8.484

24. Pawar R, Gulve N, Nehete A, Dhope S, Deore D, Chinglembi $\mathrm{N}$. Examination of the temporomandibular joint-a review. $J$ Appl Dent Med Sci. 2016;2:1.

25. Cameron MH. Physical agents in rehabilitation (an evidencebased approach to practice). $5^{\text {th }}$ ed. Philadelphia: Saunders; 2017.

26. Hertling D. Management of common musculoskeletal disorders. $4^{\text {th }}$ ed. Philadelphia: Lippincott Williams \& Wilkins; 2006.

27. Shirai M, Kawai N, Hichijo N, Watanabe M, Mori H, Mitsui $\mathrm{SN}$, et al. Effects of gum chewing exercise on maximum bite force according to facial morphology. Clin Exp Dent Res. 
2018;4(2):48-51. PMID: 29744215 DOI: 10.1002/cre2.102

28. Madani AS, Mirmortazavi A. Comparison of three treatment options for painful temporomandibular joint clicking. $J$ Oral Sci. 2011;53(3):349-54. PMID: 21959663

29. Khuman R, Chavda D, Surbala L, Chaudhary E, Bhatt U,
Nambi G. Physical therapy in temporomandibular dysfunction following maxillo-mandibular fixation in subcondylar mandibular fracture-a single case study. Int $J$ Health Sci Res. 2013;3(9):45-55. 